\title{
Disease flares, damage accrual and survival in ANCA-associated vasculitis in Western Australia
}

Background: ANCA-associated vasculitis (AAV) is a potentially life-threatening condition for which limited Australian data are available. We investigated the clinical and epidemiological outcomes of AAV in the last decade in a tertiary setting in Western Australia (WA).

Methods and findings: A single centre observational study of patients fulfilling classification criteria for granulomatous polyangiitis (GPA), eosinophilic polyangiitis (EGPA) and microscopic polyangiitis (MPA) between 2007-2016. Clinical data collected at baseline, flares and last follow-up included disease activity (BVAS), treatment regimen and damage (VDI) scores. Non-parametric tests and Kaplan Meyer survival estimates were used in analysis.

Results: During the study period there were 54 incident and 13 prevalent cases for an annual AAV incidence rate of $10.1 /$ million (95\% Cl: 7.6-13.8) with rates for GPA $(n=35)$, MPA $(n=12)$ and EGPA $(n=7)$ at $6.5,2.2$ and 1.3 per million respectively. Point prevalence per 1/1/2016 was 116.7/million. Age and gender distribution were similar between subtypes, but baseline BVAS scores were significantly higher in MPA patients ( 16 vs 10 vs $10, p<0.01$ ). The relapse rate ( 0.4 per patient-year) was equivalent across AAV subtypes with less than $30 \%$ of patients in remission and damage accrual present in $93 \%$ at last followup. Patient survival (93.2\% at 5 years) was negatively impacted especially by damage accrual with VDI $>3$.

Conclusion: The incidence of especially GPA in WA is lower than in surrounding regions. Although current management of AAV leads to good short-term survival, it associates with frequent development of organ damage, which impacts on survival.

Keywords: vasculitis • Australia • ANCA • MPO • PR3

\section{Introduction}

The Anti-Neutrophil Cytoplasmic Antibody (ANCA) associated vasculitides (AAV) are uncommon but serious diseases with a variable clinical and serological presentation across geographic regions [1-3]. Two studies from Eastern and South Australia have found the incidence of AAV/GPA in Australia as similar to Europe [4,5]. Western Australia (WA) is more than $2500 \mathrm{~km}$ distant from these areas, has a different environment and younger population, but clinical-epidemiological data for AAV in WA are not available. In addition, since these earlier studies were performed a decade ago, other drugs have been found to be equally effective but less toxic than Cyclophosphamide for AAV [6,7]. Therapeutic schedules for AAV now aim to maximise rate of remission induction and maintenance while reducing irreversible damage accumulation and side effects [8]. Based on the above considerations, we investigated the incidence, prevalence, subtype presentation and outcome of AAV (granulomatous polyangiitis
(GPA), eosinophilic polyangiitis (EGPA) and microscopic polyangiitis (MPA) in Western Australia over the last decade.

We included all adult AAV patients followed in a tertiary referral centre in Western Australia in a retrospective observational study over the period January 2007 to July 2016. Patients were identified through electronic hospital inpatient and outpatient record search (ICD10AM codes M31.1, M31.3, M31.4, M31.7, M31.8). We included only patients $\geq 18$ years with clinical condition/diagnosis compatible with AAV and residing in the catchment area for whom disease could be classified following the validated European Medicines Agency (EMA) algorithm [9], which sequentially applies ACR criteria [10,11], Lanham criteria [12] and CHCC definitions [13]. An additional list, not captured by the above search strategy, was obtained from a search of the hospital-shared drive for outpatient clinic letters using the search "vasculitis" and through various specialities

\section{Materials and methods}

Shereen Paramalingam*, Warren Raymond, Chanakya Sharma, Gursharan Dogra, Andrew Mclean-Tooke \& Johannes Nossent

Department of Rheumatology, Royal Perth Hospital, Perth, WA, Australia

*Author for correspondence: pshereen81@ yahoo.com 
(immunology, rheumatology, nephrology) that frequently manage AAV. Twelve patients with non-classifiable disease or residing outside the catchment area were excluded from further analysis. The study was categorised as a minimal risk study by Ethics and Quality Improvement Committee and did not require informed consent (GEKO no. 11342).

Data recorded on pre-defined proformas included demographics with clinical data collected at baseline, subsequent visits and at the last recorded follow-up. Data included gender, age, country of birth and residential postcode, date of AAV diagnosis, AAV sub-type and disease activity by Birmingham Vasculitis Score (BVAS) version 3 [14], ANCA pattern and titre for IF-ANCA (normal $<3 \mathrm{U}$ ) and anti- PR3 and anti-MPO antibodies by ELISA (normal $<7$ $\mathrm{U})$ and initial treatment. We also recorded the time since onset, number and severity of relapses defined as any increased BVAS score leading to escalation of therapy, organ damage score by Vasculitis Damage Index (VDI) [15], and occurrence of comorbid conditions (classified as infections, adverse reactions to drugs, malignancy and osteoporosis). Patients with clinical and serological disease quiescence with daily prednisolone dose $<7.5 \mathrm{mg}$ at last visit were considered to be in remission. Mortality data was obtained from electronic health databases and/or medical records.

Comparison of frequencies or numerical values across groups was performed with nonparametric methods, while Kaplan-Meier curves were used for survival analysis across subgroups and compared by Log-rank testing. IBM SPSS version 24 was used for statistical analysis with resulting $\mathrm{p}$ values $<0.05$ considered significant. The incidence and prevalence estimates are based on the adult population residing in the catchment area of the North Metropolitan Health Service ( $n=540.000,28 \%$ of WA population) for which our centre serves as the referral hospital for Immunology, Rheumatology and Nephrology (http://www.nmhs.health.wa.gov.au).

\section{Results}

There were 54 incident cases of AAV during the study period with 4 deaths occurring in the full cohort $(n=67)$. The annual incidence rate for all AAV was 10.1/million (95\% CI: 7.6-13.8) with incidence for GPA $(n=35)$ at .5/million $(95 \% \mathrm{CI}$ : 4.6-8.9), for MPA $(n=12)$ at $2.2 /$ million $(95 \%$ CI: $1.3-2.8)$ and for EGPA $(n=7)$ at $1.3 /$ million (95\% CI: 0.6-2.6). The point prevalence per $1 / 1 / 2016$ for all AAV was $116.7 /$ million $(95 \%$ CI: 90.4-148.3), for GPA 0.3/million (95\% CI: 50.5-95.6), for MPA 22.2/million (95\% CI: 12.0-37.8) and for EGPA 20.4/million (95\% CI: 10.7-35.2).

Age and gender distribution did not differ across AAV subtypes and while Caucasian patients presented all AAV subtypes, GPA was not observed in Asian patients (Table 1). Neuropathies were significant less frequent in GPA patients $(\mathrm{p}<0.05)$, with renal involvement more frequent in MPA and pulmonary disease as frequent in MPA as in EGPA (Figure 1) with baseline BVAS scores significantly higher in MPA patients (Table 1). Seven patients (10\%)

Table 1. Patient characteristics by AAV subtype. Figure indicates median (IQR) or number in $\%$.

\begin{tabular}{|l|l|l|l|l|l|l|}
\hline Age at Diagnosis (years) & EGPA (n=11) & GPA (n=42) & MPA (n=14) & Total & p value \\
\hline Male gender & $7(63.6)$ & $22(52.4)$ & $5(35.7)$ & $34(50.7)$ & NS \\
\hline Ethnicity & $0(0.0)$ & $1(2.4)$ & $0(0.0)$ & $1(1.5)$ & $<0.01$ \\
\hline African & $3(27.3)$ & $0(0.0)$ & $3(21.4)$ & $6(9.0)$ & \\
\hline Asian & $8(72.7)$ & $39(92.9)$ & $11(78.6)$ & $58(86.6)$ & \\
\hline Caucasian & $0(0.0)$ & $2(4.8)$ & $0(0.0)$ & $2(3.0)$ & \\
\hline Middle East & \multicolumn{5}{|l|}{} \\
\hline Classification Criteria & $8(72.7)$ & $29(69.0)$ & $2(14.3)$ & $39(58.2)$ & $<0.01$ \\
\hline ACR & $2(18.2)$ & $4(9.5)$ & $8(57.1)$ & $14(20.9)$ & \\
\hline CHCC & $1(9.1)$ & $9(21.4)$ & $4(28.6)$ & $14(20.9)$ & \\
\hline EMEA & \multicolumn{5}{|l|}{} \\
\hline IF-ANCA pattern & $1(9.1)$ & $25(59.5)$ & $2(14.3)$ & $28(41.8)$ & $<0.01$ \\
\hline C-ANCA & $1(9.1)$ & $4(9.5)$ & $5(35.6)$ & $10(14.9)$ & \\
\hline Indeterminate & \multicolumn{5}{|l|}{} \\
\hline
\end{tabular}




\begin{tabular}{|l|l|l|l|l|l|}
\hline P-ANCA & $4(36.4)$ & $5(11.9)$ & $6(42.9)$ & $15(22.4)$ & \\
\hline ANCA ELISA & $8(72.7)$ & $9(21.4)$ & $12(85.7)$ & $29(43.3)$ & $<0.01$ \\
\hline Anti-MPO & $0(0.0)$ & $28(66.7)$ & $1(7.1)$ & $29(43.3)$ & \\
\hline Anti-PR3 & $53(7-188.5)$ & $50(16-144)$ & $216(37-580)$ & $54.5(17-216)$ & \\
\hline Anti-MPO/PR3 titre (IU) & $4(63.6)$ & $33(78.6)$ & $11(78.6)$ & $51(76.1)$ & NS \\
\hline Biopsy evidence vasculitis & $10(5-12)$ & $10(6-16)$ & $19(17-24$ & $11(6-180)$ & $<0.01$ \\
\hline BVAS Score & $\begin{array}{l}\text { ACR: American College of Rheumatology; CHCC: Chapel Hill Consensus Classification; BVAS: Birmingham Vasculitis } \\
\text { Activity Score; NS: Non significant }\end{array}$ \\
\hline
\end{tabular}

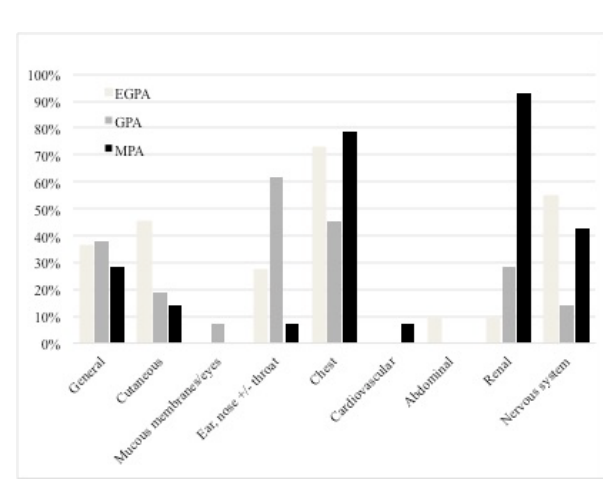

Figure 1. The distribution of organ involvement at baseline according to BVAS definitions across the AAV subtypes.

had weakly positive IF ANCA but no detectable anti-PR3 or MPO antibodies. MPA patients had the highest frequency $(86 \%)$ and titre of MPO ANCA ( $p<0.01$ for trend).

Induction therapy was with oral corticosteroids (85\%; given as IV pulse in $19.4 \%$ ), combined with cyclophosphamide $(49.2 \%)$ or other cytotoxic drugs (37\%). Two patients were lost to follow-up and during a median follow-up of 4 years, 45/65 patients (69.2\%) experienced 106 disease flares (range 1-8 per patient). There was no significant difference in the proportion of EGPA $(\mathrm{n}=8,80 \%)$, GPA $(\mathrm{n}=31,75.6 \%)$ and MPA $(\mathrm{n}=6$, $50 \%)$ patients experiencing a flare $(\mathrm{p}=0.182)$, nor the number of flares $(\mathrm{p}=0.34)$ nor the time to first flare $(\mathrm{p}=0.59)$. Complications occurred in 34 patients including severe infections (32.4\%), adverse drug reactions $(17.8 \%)$, cardiovascular events (7.4\%) and osteoporosis/fractures (5.9\%) and were as frequent in MPA $(\mathrm{n}=7,8.3 \%)$ as in GPA $(n=21,51.2 \%)$ and EGPA patients $(n=4$, $40 \%)(\mathrm{p}=0.21)$.

More GPA patients $(\mathrm{n}=12,29.3 \%)$ than MPA $(\mathrm{n}=2,16.7 \%)$ and EGPA $(0 \%)(\mathrm{p}=0.23)$ patients were in remission at last follow-up when only four patients $(6.5 \%)$ remained free of organ damage. The median VDI score at last visit was 3 (range 1-6) with no significant differences between AAV subtypes ( $\mathrm{p}=0.52)$. ENT damage was more frequent in GPA $(71.4 \%, \mathrm{p}=0.014)$, renal damage in MPA $(57.1 \%, \mathrm{p}=0.015)$ and neuropsychiatric damage in EGPA (45.5\%, $\mathrm{p}=0.03)$, while seven $(11.4 \%)$ patients developed a new malignancy (Table 2).

Overall patient survival at 1 year was $98.5 \%$ (SD $\pm 1.5 \%)$ and at 5 years $93.2 \%(\mathrm{SD} \pm 3.9 \%)$, was best for EGPA patients and worst for MPA patients (Figure 2A) (statistically not significantly different) with no influence for gender $(\mathrm{p}=0.8)$, presence of PR3 or MPO ANCA $(p=0.28)$ or ever use of cyclophosphamide $(\mathrm{p}=0.4)$. However, severe organ damage $(\mathrm{VDI}>3)$ was a predictor for poor survival (Figure 2B).

\section{Discussion}

This study found a lower incidence of especially GPA in Western Australia compared to other areas in the Southern hemisphere and demonstrates that while current management has further improved survival for AAV patients over the last decade, there is a pressing need to reduce the large burden of comorbidity.

The GPA incidence in WA (6.5/million) traces towards the lower end of rates reported from other areas in the Southern hemisphere where incidence varies between 5.8 (North Island NZ) and 25 (South Island NZ) (16) with midway rates reported for Eastern (8.8) and South Australia (11.2). Discrepancies in GPA incidence rates have been reported internationally [3] although the reasons for this are not fully understood. Perth in WA lies at 31.9 degrees latitude and based on the reported existence of a North-South gradient for GPA in this region [16] a higher incidence would be expected. Methodological differences between this and earlier studies may partly explain this as we studied AAV in a later time period and used the EMEA algorithm for classification, which was unavailable prior to 2005. Also, we did not include ANCA negative patients with PAN or patients under the age of 


\begin{tabular}{|c|c|c|c|c|c|c|c|c|}
\hline \multirow{2}{*}{ VDI categories } & \multicolumn{2}{|c|}{ EGPA $(n=11)$} & \multicolumn{2}{|c|}{ GPA $(n=42)$} & \multicolumn{2}{|c|}{ MPA $(n=14)$} & \multirow{2}{*}{\begin{tabular}{|c|} 
Chi-2 \\
p value
\end{tabular}} & \multirow[t]{2}{*}{ KW } \\
\hline & n (\%) & VDI & n (\%) & VDI & n (\%) & VDI & & \\
\hline Musculoskeletal & $5(45.5)$ & $1(1,2)$ & $8(19.0)$ & $3.5(1.5,4)$ & $3(21.4)$ & $4(2,5)$ & 0.182 & 0.242 \\
\hline Skin & $1(9.1)$ & $2(2,2)$ & $3(7.1)$ & $1(1,2)$ & $2(14.3)$ & $1(1,1)$ & 0.72 & 0.657 \\
\hline Eyes & $2(18.2)$ & $1.5(1,2)$ & $6(14.3)$ & $4(1,4)$ & $0(0.0)$ & - & 0.283 & 0.327 \\
\hline ENT & $3(27.3)$ & $2(1,2)$ & $30(71.4)$ & $2(1,4)$ & $4(28.6)$ & $1(1,1.5)$ & 0.003 & 0.003 \\
\hline Pulmonary & $6(54.5)$ & $2(2,2)$ & $17(40.5)$ & $2(1,3)$ & $7(50.0)$ & $3(1,3)$ & 0.64 & 0.283 \\
\hline CVD & $0(0.0)$ & - & $3(7.1)$ & $5(1,11)$ & $2(14.3)$ & $2.5(2,3)$ & 0.399 & 0.364 \\
\hline PVD & $0(0.0)$ & - & $2(4.8)$ & $2.5(1,4)$ & $0(0.0)$ & - & 0.541 & 0.58 \\
\hline Gastrointestinal & $1(9.1)$ & $1(1,1)$ & $0(0.0)$ & -- & $1(7.1)$ & $1(1,1)$ & 0.17 & 0.147 \\
\hline Renal & $1(9.1)$ & $1(1,1)$ & $10(23.8)$ & $2(1,4)$ & $8(57.1)$ & $3(1.5,3)$ & 0.017 & 0.004 \\
\hline Neuropsychiatric & $5(45.5)$ & $2(2,3)$ & $10(23.8)$ & $1.5(1,2)$ & $3(21.4)$ & $1(1,1)$ & 0.031 & 0.146 \\
\hline Other & $3(27.3)$ & $1(1,2)$ & $8(19.0)$ & $1.5(1,2.5)$ & $0(0.0)$ & & 0.142 & 0.172 \\
\hline
\end{tabular}

Chi 2 refers to $p$ value for comparison of $2 \times 2$ tables for categories, KW: Kruskal Wallis derived $p$ values for comparison of numerical data.

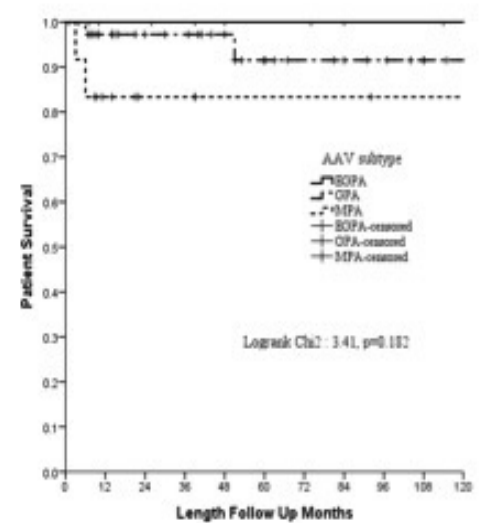

Figure 2A. Kaplan Meyer survival curves for patient by AAV subtype and organ damage (VDI>3).

eighteen as the other Australian studies [4,5]. Given the fact that incidence rates for MPA and EGPA are compatible with both the NZ and Australian data, it is also possible that our data reflect truly reflect a lower GPA incidence in WA attributable to the more extreme UV exposition in WA and/or an effect of the periodicity for GPA [17-19].

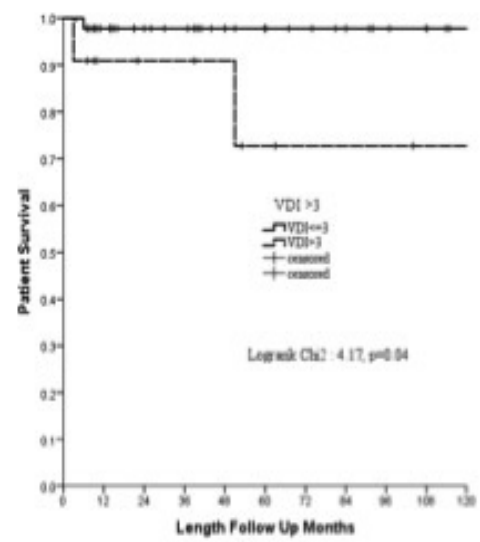

Figure 2B. Kaplan Meyer survival curves for patient by AAV subtype and accrual.

The clinical presentation of AAV in WA is largely unremarkable in terms of age and gender and organ involvement, but there was a notable absence of GPA in Asian patients, who all presented with anti-MPO ANCA. This fits well with low rate of GPA within the clinical spectrum of AAV reported in Asia [20]. 
Mortality risk amongst AAV patients are generally higher compared to the general population [21]. Patient survival at 1 year $(98.5 \% \pm 1.5 \%)$ and 5 years $(93.2 \% \pm 3.9 \%)$ was better in our study compared to more recent reports from New Zealand and Norway [1,22]. Mortality was in contrast not significantly influenced by AAV subtype or ANCA specificity. Despite this improved short-term life expectancy, we found a significant burden of infections other co-morbidities in surviving AAV patients. Few patients remained free of organ damage with high ENT damage accrual damage seen in GPA, renal damage in MPA and neuropathy in EGPA, all consistent with previous reports $[1,23]$. As VDI scores represent the combined effects of disease and drug effects, this clearly indicates that current therapy still leaves too many patients with significant residual complications. Furthermore, as such severe damage also associated with poorer survival in this study, new strategies to prevent organ damage are sorely needed.

The limitations of this study relate to the retrospective observational cohort design for a rare disease in a single catchment area. Despite being a public referral centre for the area it is possible that we may have missed patients preferring private health care and the epidemiological characteristics in this study should therefore be considered minimum estimates. On the other hand, the fact that patients were followed in a comprehensive fashion at a single centre with multidisciplinary care strengthens the results through consistency in patient management and validating outcomes.

The incidence of especially GPA in WA is lower than in surrounding regions. Although current management of AAV leads to good short-term survival, it associates with frequent development of organ damage, which impacts on survival.

\section{Acknowledgement}

Sir Charles Gairdner medical records staff who provided assistance and support throughout the course of this study.

\section{Conflict of Interest}

The authors have no conflict of interest to report regarding this study.

\section{References}

1. Khan AR, Chapman PT, Stamp LK et al. Wegener's granulomatosis: treatment and survival characteristics in a high-prevalence southern hemisphere region. Intern. Med. J. 42(4), e23-e26 (2012).
2. Lionaki S, Blyth ER, Hogan SL et al. Classification of antineutrophil cytoplasmic autoantibody vasculitides: the role of antineutrophil cytoplasmic autoantibody specificity for myeloperoxidase or proteinase 3 in disease recognition and prognosis. Arthritis. Rheum. 64(10), 3452-3462 (2012).

3. Watts RA, Mahr A, Mohammad AJ et al. Classification, epidemiology and clinical subgrouping of antineutrophil cytoplasmic antibody (ANCA)-associated vasculitis. Nephrol. Dial. Transplant. 30 (Suppl 1), i14-22 (2015).

4. Ormerod AS, Cook MC. Epidemiology of primary systemic vasculitis in the Australian Capital Territory and south-eastern New South Wales. Intern. Med. J. 38(11), 816-823 (2008).

5. Hissaria P, Cai FZ, Ahern M et al. Wegener's granulomatosis: epidemiological and clinical features in a South Australian study. Intern. Med. J. 38(10), 776-780 (2008).

6. Yates M, Watts R, Bajema I et al. Validation of the EULAR/ERA-EDTA recommendations for the management of ANCA-associated vasculitis by disease content experts. RMD. Open. 3(1), e000449 (2017)

7. van Daalen EE, Rizzo R, Kronbichler A, Wolterbeek $\mathrm{R}$ et al. Effect of rituximab on malignancy risk in patients with ANCAassociated vasculitis. Ann. Rheum. Dis. 76(6), 1064-1069 (2017).

8. Jones RB, Hiemstra TF, Ballarin $\mathrm{J}$ et al. Mycophenolate mofetil versus cyclophosphamide for remission induction in ANCA-associated vasculitis: a randomised, non-inferiority trial. Ann. Rheum. Dis. (2019).

9. Watts R, Lane S, Hanslik T, Hauser T et al. Development and validation of a consensus methodology for the classification of the ANCAassociated vasculitides and polyarteritis nodosa for epidemiological studies. Ann. Rheum. Dis. 66(2), 222-227 (2007).

10. Leavitt RY, Fauci AS, Bloch DA et al. The American College of Rheumatology 1990 criteria for the classification of Wegener's granulomatosis. Arthritis. Rheum. 33(8), 1101-1107 (1990).

11. Masi AT, Hunder GG, Lie JT et al. The American College of Rheumatology 1990 criteria for the classification of Churg-Strauss syndrome (allergic granulomatosis and angiitis). Arthritis. Rheum. 33(8), 1094-1100 (1990).

12. Lanham JG, Elkon KB, Pusey CD et al. Systemic vasculitis with asthma and eosinophilia: a clinical approach to the Churg-Strauss syndrome. Medicine (Baltimore). 63(2), 65-81 (1984).

13. Jennette JC, Falk RJ, Bacon PA et al. 2012 revised International Chapel Hill Consensus Conference Nomenclature of Vasculitides. Arthritis. Rheum. 
65(1), 1-11 (2013).

14. Mukhtyar C, Lee R, Brown D et al. Modification and validation of the Birmingham Vasculitis Activity Score (version 3). Ann. Rheum. Dis. 68(12), 1827-1832 (2009).

15. Exley AR, Bacon PA, Luqmani RA et al. Development and initial validation of the Vasculitis Damage Index for the standardized clinical assessment of damage in the systemic vasculitides. Arthritis. Rheum. 40(2), 371-380 (1997).

16. O’Donnell JL, Stevanovic VR, Frampton C et al. Wegener's granulomatosis in New Zealand: evidence for a latitude-dependent incidence gradient. Intern. Med. J. 37(4), 242-246 (2007).

17. Gatenby PA. Anti-neutrophil cytoplasmic antibody-associated systemic vasculitis: nature or nurture? Intern. Med. J. 42(9), 1066-1067 (2012).

18. Gies P. Capital city maximum UV levels by month. Melbourne, Australia (2013).
19. Yates M, Watts R. ANCA-associated vasculitis. Clin. Med. (Lond). 17(1), 60-64 (2017).

20. Fujimoto S, Watts RA, Kobayashi $S$ et al. Comparison of the epidemiology of antineutrophil cytoplasmic antibody-associated vasculitis between Japan and the U.K. Rheumatology (Oxford). 50(10), 1916-1920 (2011).

21. Tan JA, Dehghan N, Chen W et al. Mortality in ANCA-associated vasculitis: ameta-analysis of observational studies. Ann. Rheum. Dis. 76(9), 1566-1574 (2017)

22. Bjorneklett R, Solbakken V, Bostad L et al. Exploring sex-specific differences in the presentation and outcomes of ANCA-associated vasculitis: a nationwide registry-based cohort study. Int. Urol. Nephrol. 50(7), 1311-1318 (2018).

23. Draibe J, Rodo X, Fulladosa X et al. Seasonal variations in the onset of positive and negative renal ANCA-associated vasculitis in Spain. Clin. Kidney. J. 11(4), 468-473 (2018). 